\title{
The role of attention in illusory conjunctions
}

\author{
YEHOSHUA TSAL, NACHSHON MEIRAN, and NILLI LAVIE \\ Tel Aviv University, Tel Aviv, Israel
}

\begin{abstract}
In five experiments, we investigated the effects of attention on illusory conjunctions formed between features of unrelated objects. The first three experiments used a weak manipulation of attention and found that illusory conjunctions formed either among features receiving high attentional priority or among features receiving low attentional priority were not more frequent than were conjunctions formed between mixed features of different attentional priority. The last two experiments used a strong manipulation of attention and failed to reveal any evidence of true illusory conjunctions. The results are inconsistent with the feature-integration theory, which predicts that when attention is focused on a subset of items, illusory conjunctions ought to occur within and outside of the attended subset, but not between the attended and unattended items.
\end{abstract}

The feature-integration theory (Treisman, 1985; Treisman \& Gelade, 1980; Treisman \& Paterson, 1984; Treisman \& Souther, 1986) proposes that features of objects are initially represented in independent specialized maps and are subsequently unified into objects with the aid of focal attention. Features are thus preattentively freefloating, and the serial scan of attention over the visual field "glues" features that occupy the same locations into objects. The phenomenon of illusory conjunctions, first established by Treisman and Schmidt (1982), has provided a major source of support for the feature-integration theory since, unlike other findings, it is based on a direct manipulation of attention (Briand \& Klein, 1987; Prinzmetal, Presti, \& Posner, 1986). Treisman and Schmidt presented brief displays containing two peripheral digits and several central figures. Subjects were instructed to report first the digits and then the figures. This manipulation was intended to direct attention to the digits during display presentation so that the central figures would be processed with minimal attention. The first four experiments of Treisman and Schmidt's study clearly demonstrated the phenomenon of illusory conjunctions-namely, the tendency to combine features (e.g., of color and shape) of different figures into a single reported figure. In the fifth experiment, the digits were eliminated from the display, and the subjects were asked to attend to the central figures. The results indicated that the number of illusory conjunctions was reduced to that of corresponding feature errors, and Treisman and Schmidt concluded that attention integrates features into unified objects, thus preventing illusory conjunctions. It is quite possible, however, that, in the fifth experiment, illusory conjunctions were eliminated because the report of the figures was no longer

We are grateful to Ray Klein and an anonymous reviewer for their comments on an earlier draft of this paper. Requests for reprints should be addressed to Yehoshua Tsal, Department of Psychology, Tel Aviv University, Ramat Aviv 69978, Israel.

-Accepted by previous editor. Charles W. Eriksen delayed by the report of the digits, thus not allowing for sufficient time for the correctly integrated features to disintegrate and form illusory conjunctions (Tsal, 1989).

Two major problems are associated with the paradigm of illusory conjunctions that are apparent in Treisman and Schmidt's (1982) study as well as in several subsequent studies conducted by other investigators. First, as suggested above, this paradigm is susceptible to memorial influences. Thus, the observed illusory conjunctions need not reflect an initial stage of feature registration but rather the failure of memory to hold together features previously represented as whole figures. Second, the manipulation of attention in these studies has not been sufficiently strong to produce completely attended and unattended stimuli. Hence, the observed illusory conjunctions may result from locational uncertainty associated with the act of spreading, shifting, or redirecting attention during stimulus processing.

In the present experiments, attended and unattended figures were included in the same displays. This design reduced the confounding influence of memorial processes and allowed for a direct comparison between several types of illusory conjunctions. Those formed between features of high attentional priority (HAP conjunctions), those formed between features receiving low attentional priority (LAP conjunctions), and those formed between a feature of an HAP item and an LAP item (mixed conjunctions). In Experiments 1 and 2, we used a weak manipulation of attention, similar to that of Treisman and Schmidt (1982), in which LAP figures were flanked by HAP ones. In Experiment 3, we used a somewhat stronger manipulation of attention, based on the cost-benefit method (Posner, 1980) and similar to the procedure of Prinzmetal, Presti, and Posner (1986). In this experiment, the location of a target was correctly cued on most of the trials and its shape could erroneously conjoin with the color of either a near HAP distractor or a far LAP distractor. In Experiments 4 and 5, we used a strong manipulation of attention based on a variation of the cost-benefit method. Here, the target location was correctly cued on all trials, 
and thus the location of the near distractor was always attended whereas the location of the far distractor was always unattended.

Treisman and Schmidt (1982) did not fully specify how illusory conjunctions would be influenced by various manipulations of attention. They have stated that "the theory predicts that when attention is focused on a subset of presented items, illusory conjunctions should be formed either within the attended subset or outside it, but not between the attended and unattended items" (p. 118). The emphasis on the absence of these mixed conjunctions derives from the presumed nature of the operation of focal attention that distinguishes features inside and outside the attentional spotlight. The present design enabled us to assess whether illusory conjunctions occur under various manipulations of attention and whether mixed conjunctions are indeed less likely to occur than are either attended or unattended conjunctions.

\section{EXPERIMENT 1}

In Experiment 1, we presented displays similar to those used in Experiments 1 and 2 of Treisman and Schmidt (1982). Each display consisted of three central colored letters and two peripheral colored digits. Subjects were instructed to first report the digits and then the shape and color of the letters. Thus, the digits were defined as items receiving HAP, and the letters were defined as those receiving LAP. The major variation from Treisman and Schmidt's study was that the digits themselves were also colored, thus allowing these HAP colors to perceptually migrate to the central LAP figures. The major purpose of the experiment was to investigate whether, contrary to predictions of the feature-integration theory, such migrations would occur, hence producing mixed illusory conjunctions between HAP digit colors and LAP letter shapes.

\section{Method}

Subjects. The subjects were 9 undergraduates from Tel Aviv University who participated to fulfill a course requirement. All had normal or corrected-to-normal vision.

Stimuli. The stimuli were presented in a two-field Gerbrands tachistoscope. Each display consisted of three colored letters arranged in a row between two colored digits. Each letter subtended a visual angle of $0.7^{\circ}$ horizontally and $1^{\circ}$ vertically. The contourto-contour distance was $0.95^{\circ}$ between adjacent letters and $1.5^{\circ}$ between a digit and an adjacent letter. The row of letters subtended $4^{\circ}$, and the entire row, including the digits, subtended $8^{\circ}$ of visual angle. The letters were randomly selected from the following set: $\mathrm{N}, \mathrm{O}, \mathrm{S}, \mathrm{T}$, and $\mathrm{X}$, with the constraint that no letter was repeated in a display. Two different digits were randomly selected from 1 to 9 for each display. The colors of the letters and digits were randomly selected from the following set of colors: red, orange, green, blue, black, gray, and brown. The colors were randomly paired with the various letters and digits. In 40 displays, all five colors within a display were different. Only these displays were used in the analyses. Ten additional displays contained a single color repetition between two letters, between the two digits, or between a letter and a digit. These were control displays whose purpose was to eliminate the possibility that once a digit color was perceived, its absence could be inferred in the letters. These displays were randomly intermixed with the remaining ones but were excluded from the analyses.
Procedure. Prior to the experiment, the subjects were shown a few display cards and were asked to name the colors in order to insure that they could distinguish among them. The subjects were instructed to report, first, the two digits by combining them into one two-digit number, and then to report the names and colors of any letters they had seen. The importance of the primary digit report task was emphasized, and feedback was given if the responses were incorrect. Furthermore, the subjects were encouraged to provide object responses whenever possible. This instruction was added after observing, in a pilot experiment, that subjects tended to report individual features (e.g., T, red) rather than objects (e.g., a red $\mathrm{T}$ ). Each display was presented for $160 \mathrm{msec}$; it was preceded by a central white display fixation point (fixed on the tachistoscope) on a dark background, and was followed by a 200 -msec visual noise mask that covered the entire stimulus field. The set of $\mathbf{5 0}$ cards was presented twice to each subject. This block of 100 trials was preceded by 20 practice trials for which the exposure duration was set at 180 msec.

\section{Results and Discussion}

Analyses were carried out on trials in which both digits were correctly reported (75\% of the trials). Table 1 shows the mean numbers per trial of correct responses and various error types. For example, the value of .253 mean correct object reports reflects .084 overall accuracy since there were three letters presented in each display $(.253 / 3=.084)$. Of major interest was the assessment of LAP conjunctions and mixed conjunctions. We followed Treisman and Schmidt's (1982) rationale in analyzing the results. Since a certain proportion of reported illusory conjunctions could be attributed to guessing, illusory conjunctions were compared with corresponding feature errors that served as an estimate for these guessing responses. The number of LAP conjunctions (.360) was compared with the number of reported letters of which one feature (either color or shape) appeared in the display $(.084+.162=.246)$ and the other did not. Since the color and shape of any given letter could be replaced with any other two presented letter colors and shapes and two nonpresented colors and shapes, pure guessing would

Table 1

Mean Numbers per Trial of Response Types in Experiment 1

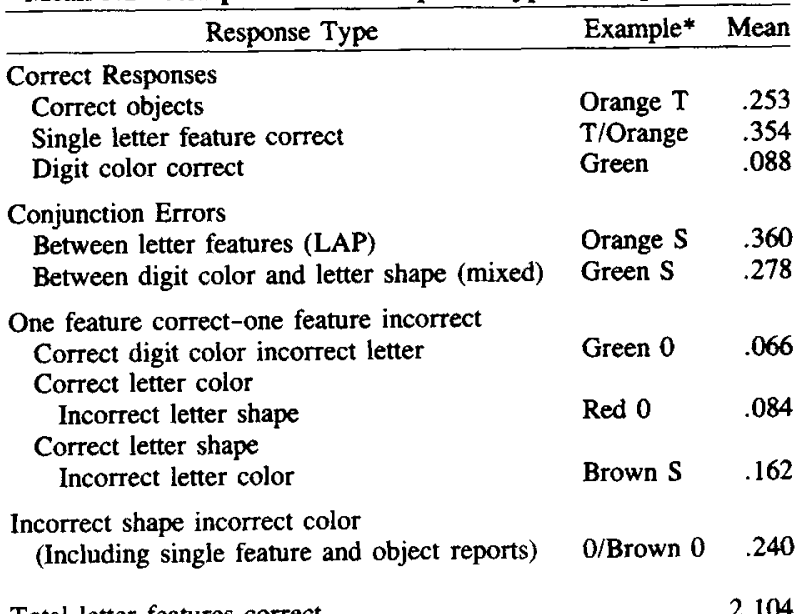

Total letter features correct

*Examples refer to the following display: green 2 ; red $S$; orange $T$; gray $X$; black 4 . 
produce an equal number of the two compared response types. Thus, LAP conjunctions in excess of these corresponding feature errors would reflect true illusory conjunctions. The number of mixed conjunctions was compared with the number of reported letter shapes, with the colors not presented in the display. The color of any given letter should be equally likely to be replaced with the two colors of the digits and the two colors not presented in the display. Hence, mixed conjunctions in excess of the above corresponding feature errors would reflect true illusory conjunctions. Note that the mixed and LAP illusory conjunctions could not be compared in this experiment, since the former involved the substitutions of colors only, whereas the latter involved the substitutions of both colors and shapes. LAP conjunctions (.360) were more frequent than were reported letters in which one feature (either shape or color) was correct and the other did not appear in the display $[.084+.162=.246 ; t(8)=4.91$, $p<.002$ ]. This finding replicates Treisman and Schmidt's main finding of illusory conjunctions between features of central letters. However, mixed conjunctions $(.278)$ were more frequent than were reported letters in which the shape was correct and the color did not appear in the display $[.162 ; t(8)=2.03, p<.05]$. This finding is not predicted by the feature-integration theory since it shows that colors of HAP digits could perceptually migrate to form illusory conjunctions with LAP shapes. It should be noted, however, that this is not a strong test of the theory since the letters and digits were not completely attended and unattended, respectively, but rather varied in attentional priority.

\section{EXPERIMENT 2}

The results of Experiment 1 suggest that colors of HAP figures can perceptually migrate and form illusory conjunctions with LAP shapes. It therefore seems that attention does not eliminate illusory conjunctions. Experiment 2 was designed to test whether or not attention reduces the level of illusory conjunctions. We presented four colored outline shapes and two black digits appearing randomly inside two of the figures. The subjects were instructed to report the digits first and then the shapes and colors of all figures. Thus, the two figures containing the digits were defined as HAP figures, and the other two figures were defined as LAP figures. Three types of illusory conjunctions were possible: within HAP figures (HAP conjunctions), within LAP figures (LAP conjunc- tions), and between features of an HAP figure and an LAP figure (mixed conjunctions).

\section{Method}

Subjects. The subjects were 8 undergraduates from Tel Aviv University who participated to fulfill a course requirement. All had normal or corrected-to-normal vision.

Stimuli. Each display included a row of four outlined colored shapes that were randomly selected from the following set of shapes-triangle, square, circle, rectangle, diamond, ellipse, and hexagon-with the constraint that no shape was repeated in the display. The rectangle and ellipse were placed with their narrow base horizontal. The colors were randomly selected from the following set-red, orange, green, black, blue, brown, and light blue-with the constraint that no color was repeated in a display. The shapes and the colors were randomly paired. The longest and shortest figures subtended vertical angles of $2.6^{\circ}$ and $1.3^{\circ}$, respectively. The widest and narrowest figures subtended horizontal angles of $1.5^{\circ}$ and $1^{\circ}$, respectively. The distance between centers of adjacent figures was $2.5^{\circ}$, and the entire row of figures subtended a visual angle between $8.5^{\circ}$ and $9^{\circ}$. Two different black digits were randomly selected from 1 to 9 and were placed at the centers of two of the figures. These figures were randomly selected for each display. A digit subtended a visual angle of $0.5^{\circ}$ vertically and $0.4^{\circ}$ horizontally.

Procedure. A set of 40 display cards was prepared and presented twice to each subject. These 80 trials were preceded by 20 practice trials. Exposure duration was $200 \mathrm{msec}$ for the practice trials and $180 \mathrm{msec}$ for the experimental trials. In all other respects, the procedure was identical to that of Experiment 1.

\section{Results and Discussion}

In this experiment, we observed that some subjects tended to confuse some features substantially more than they did other features. For example, the red was frequently confused with orange, and the ellipse was frequently confused with the rectangle. This response may suggest errors of perceptibility or may be due to the large number of colors and shapes used in the experiment. Although this response introduced substantial noise into the analyses, it did not bias the results in any direction, since it had an equal effect on illusory conjunctions and on reported objects with one correct and one incorrect feature.

Analyses were performed on trials in which both digits were correctly reported $(71.25 \%$ of the trials). Table 2 shows the mean corrected numbers per trial of the various responses. As in Experiment 1, the proportions were first calculated for each subject by dividing the frequency of each response by the total number of trials in which both digits were correctly reported. Since, unlike in Experiment 1 , the total numbers of possible responses (prior probabilities) were different for the various response

Table 2

Mean Corrected Numbers per Trial of Response Types in Experiment 2

\begin{tabular}{llcccc}
\hline & $\begin{array}{c}\text { Illusory } \\
\text { Conjunctions }\end{array}$ & $\begin{array}{c}\text { Iterns with One } \\
\text { Correct and } \\
\text { Feature }\end{array}$ & $\begin{array}{c}\text { Correct Item } \\
\text { (Both Correct } \\
\text { Color \& Shape) }\end{array}$ & $\begin{array}{c}\text { Single } \\
\text { Feature } \\
\text { Correct }\end{array}$ & $\begin{array}{c}\text { Total } \\
\text { Feature } \\
\text { Correct }\end{array}$ \\
\hline High attentional priority (HAP) & $.047(2)^{*}$ & $.035(12)$ & $.301(2)$ & $.055(4)$ & $.598(4)$ \\
Low attentional priority (LAP) & $.038(2)$ & $.019(12)$ & $.061(2)$ & $.030(4)$ & $.276(4)$ \\
Mixed & $.045(8)$ & & & & \\
\hline
\end{tabular}

*Prior probabilities are given in parentheses. 
types, the above proportions were corrected by dividing them by these prior probabilities, as explained below. For each display, there were 2 possible HAP conjunctions, 2 possible LAP conjunctions, 8 possible mixed conjunctions, and 12 possible combinations of items with one correct feature and one incorrect feature (i.e., a feature included in the set but not presented in the display). To correct for unequal chance responses, the mean values were divided by these corresponding prior probabilitiesnamely, by the above total number of possible combinations for each response. These corrected proportions are presented in the table and were used in the analyses. The numbers in parentheses represent the prior probabilities by which the original proportions were divided. The total number of the correctly reported features (including single reported features, a feature of reported items with one correct and one incorrect feature, features of illusory conjunctions, and both features from correctly reported items) was greater for the HAP figures than for the LAP figures $[t(7)=8.34, p<.001]$. Thus, as intended, including the digits in two of the figures resulted in allocating more attention to these figures than to the remaining two. This finding validates the major manipulation of the present experiment.

The next analysis compared HAP and LAP conjunctions, excluding mixed conjunctions. This two-way figure type (HAP vs. LAP) $\times$ error type (illusory conjunctions vs. items with one correct and one incorrect feature) within-subject analysis of variance (ANOVA) was performed on proportions per trial divided by their corresponding prior probabilities. The only significant effect was that of error type; illusory conjunctions were more frequent than were items with one correct and one incorrect feature $[F(1,7)=5.65, p<.05]$. Thus, at least some of these conjunctions represent true illusory conjunctions between features of unrelated objects. Neither figure type $[F(1,7)=3.17]$ nor the interaction $[F(1,7)=0.18]$ was significant. Unlike the results obtained by Treisman and Schmidt (1982), in the present experiment, true illusory conjunctions (i.e., total illusory conjunctions relative to items with one correct and one incorrect feature) were equally frequent between a shape and a color of two HAP figures and between those of two LAP figures. Although it is not the major focus of the experiment, this finding also seems incongruent with the feature-integration theory. Treisman and Schmidt stated that they would expect illusory conjunctions between features of several items attended simultaneously, presumably because the correct integration of features requires a serial scan in which each item is attended individually. However, the present experiment probably necessitated serial processing of the two attended figures. These figures appeared in two of four possible positions and contained two digits that needed to be reported. The two-digit report may have required a serial scan. Moreover, since the two attended figures were nonadjacent in half of the trials, it is unlikely that attention was simultaneously focused on two remote figures appearing at unexpected locations. It therefore seems that illusory conjunctions were not reduced even when attention was probably serially focused on the two attended items.

Two comparisons were carried out to investigate the major question of this experiment-namely, whether mixed conjunctions were less frequent than either HAP or LAP conjunctions. First, a within-subject ANOVA compared the three types of illusory conjunctions and found no difference between the three $[F(2,14)=.43]$. The second comparison contrasted HAP and LAP conjunctions versus mixed conjunctions. As indicated in Table 2, the average corrected proportions of HAP and LAP conjunctions were, in fact, smaller than the corrected proportion for mixed conjunctions, but this effect was not significant. Thus, contrary to predictions of the featureintegration theory, illusory conjunctions between features of HAP and LAP items were just as likely to be formed as those between HAP features or between LAP features. Again, it should be noted that the present experiment did not provide a strong test of feature-integration theory since all items required some processing; therefore, there were no items that were completely unattended.

\section{EXPERIMENT 3}

Experiments 1 and 2 showed no evidence of any effects of attention on the magnitude of illusory conjunctions. However, it is possible that the method we used did not allow for a sufficiently strong manipulation of attention. Although the manipulation checks we employed indicated that items designated as attended received more attention than those designated as unattended, it is still possible that the latter were not fully unattended. First, the report of these unattended items was required. Second, the attentional system may not be sufficiently flexible to exclude those unattended items that were flanked by attended ones. Furthermore, the memory load in Experiments 1 and 2 was higher than in Treisman and Schmidt's (1982) study, since, in the present experiments, the subjects were instructed to report the identities and colors of either three letters or four shapes. Thus, conjunctions obtained in the present experiments may have been contaminated by memorial processes.

Experiment 3 used an entirely different method to manipulate attention. The subjects were presented with three items of different colors: A single target (either an S or an $\mathrm{X}$ ) and two distractors (Os). The near distractor appeared near the target on the same side of fixation; the far distractor appeared on the opposite side of fixation. We employed the cost-benefit paradigm (Posner, 1980): On $70 \%$ of the trials, a peripheral precue appeared near the location of the target and the near distractor, and on $30 \%$ of the trials, it appeared near the location of the far distractor. The subjects were instructed to report the shape and color of the target. Of major interest was the assessment of illusory conjunctions formed between the target shape and the color of either the near distractor (near conjunctions) or the far distractor (far conjunctions). The near 
conjunctions involved two HAP features in the valid trials and two LAP features in the invalid trials. The far conjunctions represented mixed illusory conjunctions in which one feature received high attentional priority and the other received low attentional priority. This design presents an improvement over Experiments 1 and 2 in manipulating attention. Especially in the valid trials, the item designated as a LAP item (the far distractor) was minimally attended. Its report was not required, and it occupied a location that was substantially remote from the cued target. However, the present procedure still does not insure that the items will be completely attended or unattended, since subjects may adopt a probability matching strategy in which the uncued location is attended on some of the trials (Eriksen \& Yeh, 1985; Jonides, 1983). Of major interest was determining whether, contrary to featureintegration theory, the color of this LAP distractor could form illusory conjunctions with the shape of the HAP target. Furthermore, in the present experiment, we minimized the potential contribution of memorial factors by eliminating the digits and by requiring the report of a single item only. Moreover, since some of our conclusions were based on a null effect, we substantially increased both the number of trials and the number of subjects.

\footnotetext{
Method

Subjects. The subjects were 18 undergraduates from Tel Aviv University who participated to fulfill a course requirement. All had normal or corrected-to-normal vision.

Stimuli. The stimuli were presented in a four-field Gerbrands tachistoscope. Each stimulus display consisted of three items centered on three vertices of an imaginary rectangle that was centered on the fixation point. The horizontal and vertical sides of this rectangle subtended $3.4^{\circ}$ and $1.45^{\circ}$ of visual angle, respectively. The three items were a target (either an S or an X) and two distractors (Os). The near distractor appeared either above or below the target on the same side of fixation. The far distractor appeared either horizontally across or diagonally across from the target on the opposite side of fixation. Each letter subtended a visual angle of $.35^{\circ}$ in height and $.3^{\circ}$ in width. The target and distractors in each display were of three different colors selected from the following set: pink, orange, light blue, and black. All combinations of stimulus arrangements (target identity, target and distractor positions, and color combinations) appeared equally often and in random order. The stimulus display was preceded by a black dot that precued the location of the target on $70 \%$ of the trials (valid trials) and appeared near the far distractor on $30 \%$ of the trials (invalid trials). The dot subtended a visual angle of $.2^{\circ}$ in diameter. In the valid trials, it appeared between the target and the near distractor slightly further toward the periphery. The contour-to-contour distance between the dot and the target or the near distractor was $.5^{\circ}$ of visual angle. In the invalid trials, the dot was diametrically opposite to its valid position; its contour-to-contour distance from the far distractor was $.5^{\circ}$ of visual angle.
}

Procedure. Each trial consisted of the following sequence: First a central black fixation dot appeared for $1 \mathrm{sec}$. It was immediately replaced by the dot cue for $100 \mathrm{msec}$. The cue was followed by the stimulus display whose duration was set individually for each subject. The display was followed by a 100 -msec visual noise mask that covered the entire stimulus field. The subjects were instructed to report the shape and color of the target. They were strongly encouraged to attend to the cue without moving their eyes, since the cue would indicate the location of the target on $70 \%$ of the trials. The stimulus display was first presented for $90 \mathrm{msec}$ and was gradually adjusted by checking the proportion of feature errors every 30 trials. A feature error was defined as reporting either the wrong target letter or a color that did not appear in the display as the target color. If the proportion of these errors was less than $30 \%$, exposure duration was reduced by $20 \mathrm{msec}$ for the following 30 trials; if this proportion was more than $30 \%$, it was increased by $20 \mathrm{msec}$. This procedure was continued throughout the experiment, with the constraint that exposure duration never exceeded $90 \mathrm{msec}$, to insure that the total duration of the cue and the display would be sufficiently brief to prevent effective eye movements. The mean exposure duration was $73 \mathrm{msec}$ and ranged from 50 to $90 \mathrm{msec}$. The subjects were presented with a single block of 360 trials in the same random order. The cards were randomized with the following constraints: no more than three successive trials were allowed for invalid trials, for the same target letter, or for the same target position. The experimental trials were preceded by 27 practice trials in which the stimulus duration was set at $90 \mathrm{msec}$.

\section{Results and Discussion}

The subjects failed to respond to the target on $13 \%$ of the valid trials and $19 \%$ of the invalid trials. Table 3 presents the mean numbers per trial of correct responses and various error types for valid and invalid trials. These proportions were calculated for each subject by dividing the frequency of each response by the total number of either the valid or the invalid trials in which the subject responded to the target. A distinction ought to be made between results obtained for the valid and invalid trials. In the invalid trials, it is not clear to what extent the target and the near distractor were unattended. Although the precue directed attention to the location of the far distractor, the subjects may have redirected attention to the target location upon its appearance. In the valid trials, on the other hand, the distinction between attended and unattended items was substantially stronger. The cue directed attention to the location of the target and near distractor. The location of the far distractor was remote from the target, and its report was not required. Hence, although analyses were performed on the entire set of results, the comparisons within the valid trials represent a stronger test of predictions of the feature-integration theory.

The first analysis indicated that correct objects (both correct shape and color) were reported more frequently in the valid than in the invalid trials $[t(17)=2.10, p<$

Table 3

Mean Numbers per Trial of Response Types in Experiment 3

\begin{tabular}{lccccc}
\hline & $\begin{array}{c}\text { Incorrect } \\
\text { Shape }\end{array}$ & $\begin{array}{c}\text { Correct } \\
\text { Object }\end{array}$ & $\begin{array}{c}\text { Color Intrusion } \\
\text { Correct Shape, } \\
\text { Unpresented } \\
\text { Color) }\end{array}$ & $\begin{array}{c}\text { Near Conjunction } \\
\text { (Correct Shape, } \\
\text { Color From Near } \\
\text { Distractor) }\end{array}$ & $\begin{array}{c}\text { Far Conjunction } \\
\text { (Correct Shape, } \\
\text { Color From Far } \\
\text { Distractor) }\end{array}$ \\
\hline Valid & .241 & .449 & .080 & .126 & .103 \\
Invalid & .244 & .405 & .096 & .110 & .145 \\
\hline
\end{tabular}


.030]. This comparison is, in fact, an underestimation of the strength of the present attentional manipulation. As indicated above, in the invalid trials, the target may not have been fully unattended since subjects may have redirected attention to its location.

Of major interest were the various comparisons between color intrusions, near conjunctions, and far conjunctions. Color intrusions signify reports of correct target shape with the color not presented in the display. They served as a baseline for guessing responses to which the near conjunctions and far conjunctions were each compared. Pure guessing should produce an equal magnitude of these three error types since the target color was equally likely to be confused with the color of the near distractor, the color of the far distractor, or the color not presented in the display. Thus, conjunctions in excess of these color intrusions reflect true illusory conjunctions. The near conjunctions signify illusory conjunctions of correct target shape with the color of the near distractor. For the valid trials, these two features received high attentional priority, and, for the invalid trials, these two features received low attentional priority since the precue appeared near the far distractor. The far conjunctions signify conjunctions between the target shape and the color of the far distractor. They represent mixed illusory conjunctions between an HAP feature and an LAP feature. In the valid trials, the target shape was HAP, and the color of the far distractor was LAP. In the invalid trials, the target shape was LAP, and the color of the far distractor was HAP.

The data were submitted to an overall error type (color intrusions vs. near conjunctions vs. far conjunctions) $x$ trial type (valid vs. invalid) within-subject ANOVA. The analysis revealed that all effects were significant $[F(2,34)=$ $5.73, p<.01$, for error type; $F(1,17)=9.89, p<.01$, for trial type; and $F(2,34)=3.90, p<.05$, for their interaction]. Further analyses clarified these effects. Far conjunctions were more frequent than color intrusions both in the valid trials $[F(1,17)=6.82, p<.05]$ and invalid trials $[F(1,17)=6.89, p<.05]$. Near conjunctions were more frequent than color intrusions in the valid trials $[F(1,17)=14.63, p<.005]$, but not in the invalid trials $[F(1,17)=.81]$. These findings show true mixed conjunctions between HAP and LAP features for both valid and invalid trials, and between HAP features in the valid trials, but not between LAP features in the invalid trials. A further analysis revealed that the difference between near and far conjunctions in the valid trials was not significant $[F(1,17)=2.58, p=.13]$. The featureintegration theory predicts that mixed illusory conjunctions ought not to occur at all. Not only did we obtain these conjunctions, but we found that their rate of occurrence was not significantly different from those of HAP illusory conjunctions in the valid trials. To compare the frequency of true far conjunctions between the valid and invalid trials, the data were submitted to an error type (color intrusion vs. far conjunctions) $\times$ trial type (valid vs. invalid) within-subject ANOVA. Both error type $[F(1,17)=$ $9.86, p<.01]$ and trial type $[F(1,17)=8.96, p<.01]$ were significant, but not the interaction $[F(1,17)=2.11$, $p=.16]$. The absence of a significant interaction suggests that mixed illusory conjunctions were similarly likely to occur in the valid and invalid trials. It is possible that this interaction did not reach significance because of insufficient power. The implications of this possibility are discussed below.

The present findings seem inconsistent with the featureintegration theory. Most importantly, the results for the valid trials indicate that the color of a far LAP distractor and the color of a near HAP distractor were equally likely to form an illusory conjunction with the shape of an HAP target. The results of the invalid trials also seem unsupportive of the feature-integration theory. Consider first the possibility that the target and the near distractor indeed received LAP, since the subjects did not have time to redirect attention to the target before its representation was disrupted by the visual mask. In this case, the data demonstrate mixed illusory conjunctions in the absence of LAP illusory conjunctions. Alternatively, it is possible that the subjects did succeed in effectively redirecting attention from the far distractor to the target. But mixed illusory conjunctions in this case are most clearly incompatible with the feature-integration theory. The theory predicts that the serial scan of attention over different items glues features into objects, thus preventing illusory conjunctions. Hence, illusory conjunctions between the color of the far distractor and the shape of the target that were attended serially are clearly inconsistent with the theory.

Another unexpected pattern of results emerging from the overall comparison of the present data may suggest an alternative interpretation of the relation between attention and illusory conjunctions. As is apparent from Table 3 , the comparative magnitude of near and far conjunctions is reversed in the valid and invalid trials-there was a greater proportion of near conjunctions in the valid trials, but a greater proportion of far conjunctions in the invalid trials. Although not completely supported by the statistical analyses, this tendency is quite illuminating. It shows that features near the focus of attention have a greater likelihood of becoming candidates for illusory conjunctions than do those outside the focus of attention. Thus, it seems that on those trials in which the target color was either not sufficiently processed or not correctly localized, it was replaced by the color of a distractor that was made more visible as a result of the focus of attention.

\section{EXPERIMENT 4}

Experiment 3 showed no evidence of attentional effects on the overall frequency of illusory conjunctions. It is still possible, however, that in this experiment the manipulation of attention was not sufficiently strong to produce truly attended and unattended stimuli. That is, the conventional cost-benefit paradigm may induce a strategy that strongly interferes with the intended manipulation of attention. Since on some trials the target appeared in the 
noncued location, the subjects may have distributed their attention over the entire display or, alternatively, may have adopted a probability matching strategy (Eriksen \& Yeh, 1985; Jonides, 1983) and directed their attention to the noncued location on a certain proportion of the trials. In the present experiment, the location of the target and near distractor was always valid, and the location of the far distractor was always invalid. This manipulation of a completely informative peripheral cue in conjunction with the automatic attraction of attention induced by its abrupt onset provides the strongest possible manipulation of attention that ought to distinguish between completely attended and unattended items. Feature-integration theory predicts illusory conjunctions between the shape of the target and the color of the near distractor, since they both fall within the region of the attentional "beam." The theory further predicts no illusory conjunctions between the shape of the target and the color of the far distractor, since the location of the former is attended and the location of the latter is unattended.

\section{Method}

Subjects. The subjects were 8 undergraduates fulfilling a course requirement. All had normal or corrected-to-normal vision.

Stimuli and Procedure. The stimuli and procedure were identical to those of Experiment 3, with one exception. The cue was always valid-namely, it always marked the correct location of the target. The subjects were strongly encouraged to pay attention to the cue since it always indicated the location of the subsequent target. Exposure duration was gradually adjusted, as in Experiment 3. The mean duration was $71 \mathrm{msec}$ and ranged from 50 to $90 \mathrm{msec}$.

\section{Results and Discussion}

The top row of Table 4 presents the mean number per trial of correct responses and various error types. An overall comparison among proportions of color intrusions, near conjunctions, and far conjunctions showed a significant effect $[F(2,14)=4.06, p<.05]$. Contrary to expectation, however, the results failed to show any evidence of true illusory conjunctions. Far conjunctions did not exceed color intrusions $[F(1,7)=0.5]$, and near conjunctions, in fact, tended to be fewer than color intrusions $[F(1,7)=5.2, p=.057]$. Interestingly, the proportion of far conjunctions tended to be higher than that of near conjunctions $[F(1,7)=5.45, p=.0521]$.

\section{EXPERIMENT 5}

Experiment 5 was an additional attempt to obtain illusory conjunctions under cuing conditions similar to those of Experiment 4. To facilitate the formation of illusory conjunctions, we added three additional possible colors to the display.

\section{Method}

Subjects. The subjects were 12 undergraduates fulfilling a course requirement. All had normal or corrected-to-normal vision.

Stimuli and Procedure. The stimuli and procedure were identical to those of Experiment 4, except that three colors (red, brown, and green) were added. The three different colors of the target and two distractors for each display were randomly selected from the seven possible colors. The mean adjusted duration was $77 \mathrm{msec}$ and ranged from 50 to $90 \mathrm{msec}$.

\section{Results and Discussion}

The bottom row of Table 4 presents the mean number per trial of correct responses and various error types. Since in each trial there were four possible colors not presented in the display, the proportions of color intrusions were divided by 4 , and these corrected proportions were compared with the proportions of near and far conjunctions. Table 4 suggests that the corrected proportion of color intrusions was, in fact, higher than that of either near or far conjunctions. However, an overall comparison among these three proportions showed no significant effect $[F(2,22)=1.69, p=.207]$. Thus, similar to $\mathrm{Ex}-$ periment 4 , the results failed to show any evidence of true illusory conjunctions. It is possible that this $100 \%$ validity in Experiments 4 and 5 encouraged eye movements. Rapid effective eye movements might have occurred in a small proportion of the trials presenting the cue and the display for a total duration of about $190 \mathrm{msec}$. However, such a possibility does not weaken the present conclusions, since these movements should have increased the visibility of the near distractor, thus increasing the likelihood of near conjunctions between the target and the near distractor that were both foveal and attended simultaneously.

\section{GENERAL DISCUSSION}

The present study investigated the effects of attention on illusory conjunctions with three different manipulations of attention. Experiments 1 and 2 used a weak manipulation, similar to Treisman and Schmidt (1982), in which LAP items were flanked by HAP ones. Experiment $3 \mathrm{em}-$ ployed a stronger manipulation of attention by using a variation of the cost-benefit paradigm similar to that used in Prinzmetal, Presti, and Posner (1986). The results of these three experiments failed to reveal significant effects of attention on the magnitude of illusory conjunctions; similar rates of conjunctions were obtained between features receiving HAP, between features receiving LAP, and between mixed (one HAP and one LAP) features. Experiments 4 and 5 employed a strong manipulation of attention in which the target and one distractor were always attended whereas another distractor was always unattended. These experiments failed to reveal true illusory

Table 4

Mean Numbers per Trial of Response Types in Experiments 4 and 5

\begin{tabular}{cccccc}
\hline Experiment & $\begin{array}{c}\text { Incorrect } \\
\text { Shape }\end{array}$ & $\begin{array}{c}\text { Correct } \\
\text { Object }\end{array}$ & $\begin{array}{c}\text { Color } \\
\text { Intrusion }\end{array}$ & $\begin{array}{c}\text { Near } \\
\text { Conjunction }\end{array}$ & $\begin{array}{c}\text { Far } \\
\text { Conjunction }\end{array}$ \\
\hline 4 & .054 & .600 & .125 & .097 & .124 \\
5 & .003 & .607 & .290 & .063 & .059 \\
\hline
\end{tabular}


conjunctions-namely, the rate of conjunctions did not exceed that of corresponding feature errors.

The fact that illusory conjunctions, when they occur, are insensitive to attentional manipulations is inconsistent with the feature-integration theory, which claims that attention glues features into objects and ought, therefore, to reduce the magnitude of illusory conjunctions. In spite of Treisman and Schmidt's (1982) explicit claim that conjunctions cannot be formed between attended and unattended features, Experiment 1 showed that the colors of two peripheral digits receiving HAP could perceptually migrate and wrongly combine with shapes of less attended central letters. Moreover, Experiments 2 and 3 showed that this type of mixed conjunctions is not less frequent than are conjunctions formed between two items receiving HAP or between two items receiving LAP.

It should be noted that although the present findings are inconsistent with the feature-integration theory, they are not in disagreement with much of the available evidence. Treisman and her associates devoted much of their empirical work to establishing the phenomenon of illusory conjunctions for unattended figures and investigating the nature of this phenomenon. Illusory conjunctions were studied either by diverting attention away from the figures (see, e.g., Ivry \& Prinzmetal, 1991; Treisman \& Schmidt, 1982) or by presenting a large number of figures in order to overload attention (Prinzmetal, 1981; Treisman \& Paterson, 1984). The only major attempt to show that illusory conjunctions are reduced when attention is focused on the display was performed in Experiment 5 of Treisman and Schmidt. However, there are several alternative interpretations of the results obtained in that experiment (Tsal, 1989). The results of other studies investigating the effects of attention on illusory conjunctions do not clarify the role of attention in feature integration. Briand and Klein (1987) suggested that this effect is obtained only when the items are cued peripherally but not when they are cued centrally. Duncan (1979) found that when attended and unattended items are presented in the same display, illusory conjunctions are formed between features of attended items but not between those of unattended ones. Cohen and Ivry (1989) found that illusory conjunctions between unattended items occur only when they are less than $1^{\circ}$ of visual angle apart. Houck and Hoffman (1986) showed that features of color and orientation could integrate without attention and produce the McCollough effect. This finding is of special significance, since it reflects the effects of uncontrollable processes and as such is less likely than are other findings to be contaminated by memorial processes. The examples cited above show that illusory conjunctions are influenced by a variety of factors, but they certainly do not provide uniform support to the notion that attention facilitates feature integration. As argued in detail elsewhere (Tsal, 1989) feature-integration theory has to demonstrate a unique relationship between attention and the magnitude of illusory conjunctions. That is, this magnitude should be strictly influenced by strong attentional manipulations but not by other variables. The literature suggests that neither one of these requirements is fully met. First, there are numerous studies showing that the rate of illusory conjunctions is influenced by several variables in a manner that is incompatible with the theory. For example, the rate of conjunctions can be strongly influenced by pure cognitive factors such as the subjective organization of the items (Prinzmetal \& Keysar, 1989), as well as by linguistic factors (Fang \& Wu, 1989; Prinzmetal \& Millis-Wright, 1984; Prinzmetal, Treiman, \& Rho, 1986). For example, Prinzmetal, Treiman, and Rho showed that conjunctions of colors were less likely to occur between letters in different syllables than between letters within a syllable in words and pseudowords. These findings suggest that memorial processes can produce illusory conjunctions between previously integrated features.

Second, most of the studies investigating illusory conjunctions used weak manipulations of attention similar to our Experiments 1-3. Several studies adopted Treisman and Schmidt's (1982) procedure of asking subjects to first report peripheral digits and then to report the central letters. They found that illusory conjunctions were more likely to occur between similar than between dissimilar features (Ivry \& Prinzmetal, 1991), that illusory conjunctions among letter features occurred in a forced-choice decision task but not in a bar-probe task (Butler, Mewhort, \& Browse, 1991), and that the magnitude of illusory conjunctions was influenced by the linguistic structure and complexity of the stimuli (Fang \& Wu, 1989).

Several other studies examined illusory conjunctions with the cost-benefit paradigm similar to that used in the present Experiment 3. They presented a peripheral cue that marked the correct target location on most of the trials and obtained illusory conjunctions between color and shape (Prinzmetal, Presti, \& Posner, 1986) and between features of different letters (Briand \& Klein, 1987). Unlike in the present Experiment 3, they claimed to have shown a greater amount of conjunctions for invalid relative to valid trials. However, the specific patterns of results of these studies are not predicted by the featureintegration theory. Briand and Klein found that attention reduced the number of conjunctions when the items were cued peripherally but not when they were cued centrally. Prinzmetal, Presti, and Posner (1986) showed that attention reduced the number of conjunctions formed between items that were attended simultaneously. Moreover, as discussed in detail elsewhere (Tsal, 1989), there are alternative interpretations to the results of these studies that do not necessarily implicate attention as a determinant of illusory conjunctions.

The present Experiments 4 and 5 used a substantially stronger manipulation of attention than did the studies reviewed above and failed to reveal any evidence of true illusory conjunctions. Hence, it is possible that illusory conjunctions between attended items or between mixed items occur only with weak manipulations of attention that do not clearly distinguish between attended and unattended items. These manipulations may encourage the spreading or shifting of attention during stimulus processing, resulting in locational uncertainty. It seems that the focus- 
ing of attention on a given location prior to stimulus onset in situations that do not entail uncertainty and thus minimize attentional redirection or redistribution facilitates localization of features and thus prevents their migration to other locations. The present proposition is consistent with the view that attention facilitates the overall processing of the stimulus, enhancing the perception of its features as well as its localization. Thus, an unattended feature is unlikely to conjoin with an attended one since the system recognizes the difference in their clarity or visibility. The features of two attended items are unlikely to conjoin since they can both be independently localized within the same attended area.

\section{REFERENCES}

Briand, K. A., \& KLEIN, R. M. (1987). Is Posner's "beam" the same as Treisman's "glue"? On the relation between visual orienting and feature integration theory. Journal of Experimental Psychology: Human Perception \& Performance, 13, 228-241.

Butler, B. E., MEWhort, D. J. K., \& BrowSE, R. A. (1991). When do letter features migrate? A boundary condition for feature-integration theory. Perception \& Psychophysics, 49, 91-99.

Cohen, A., \& IVRY, R. (1989). Illusory conjunctions inside and outside the focus of attention. Joumal of Experimental Psychology: $\mathrm{Hu}$ man Perception \& Performance, 15, 650-663.

DUNCAN, J. (1979). Divided attention: The whole is more than the sum of its parts. Joumal of Experimental Psychology: Human Perception \& Performance, 5, 215-228.

ERIKSEN, C. W., \& YEH, Y. Y. (1985). Allocation of attention in the visual field. Journal of Experimental Psychology: Human Perception \& Performance, 5, 583-597.

FANG, S. P., \& WU, D. (1989). Illusory conjunctions in the perception of Chinese characters. Joumal of Experimental Psychology: Human Perception \& Performance, 15, 434-447.

HoucK, M. R., Hoffman, J. E. (1986). Conjunction of color and form without attention: Evidence from an orientation-contingent color aftereffect. Journal of Experimental Psychology: Human Perception \& Performance, 12, 186-199.
IVry, R. B., \& Prinzmetal, W. (1991). Effect of feature similarity on illusory conjunctions. Perception \& Psychophysics, 49, 105-116.

JoNIDEs, J. (1983). Further toward a model of the mind's eye's movement. Bulletin of the Psychonomic Society, 21, 247-250.

Posner, M. I. (1980). The orienting of attention. Quarterly Journal of Experimental Psychology, 32, 3-25.

Prinzmetal, W. (1981). Principles of feature integration in visual perception. Perception \& Psychophysics, 30, 330-340.

Prinzmetal, W., \& KeYsar, B. (1989). Functional theory of illusory conjunctions and neon colors. Journal of Experimental Psychology: General, 118, 165-190.

Prinzmetal, W., \& Millis-Wright, M. (1984). Cognitive and linguistic factors affect visual feature integration. Cognitive Psychology, $6,305-340$.

Prinzmetal, W., Presti, D. E., \& Posner, M. I. (1986). Does attention affect visual feature integration? Journal of Experimental Psychology: Human Perception \& Performance, 12, 361-369.

Prinzmetal, W., Treiman, R., \& RHo, C. (1986). How to see a reading unit. Journal of Memory \& Language, 25, 461-475.

Treisman, A. (1985). Preattentive processing in vision. Computer Vision, Graphics, \& Image Processing, 31, 156-177.

Treisman, A., \& Gelade, G. (1980). A feature-integration theory of attention. Cognitive Psychology, 12, 97-136.

Treisman, A. Paterson, R. (1984). Emergent features, attention and object perception. Journal of Experimental Psychology: Human Perception \& Performance, 10, 12-31.

Treisman, A., \& SchmidT, H. (1982). Illusory conjunction in the perception of objects. Cognitive Psychology, 14, 107-141.

Treisman, A., \& Souther, J. (1986). Illusory words: The role of attention and top-down constraints in conjoining letters to form words. Journal of Experimental Psychology: Human Perception \& Performance, 12, 3-17.

Tsal, Y. (1989). Do illusory conjunctions support the feature integration theory? A critical review of theory and findings. Journal of $E x$ perimental Psychology: Human Perception \& Performance, 15, 394-400.

(Manuscript received August 2, 1988; revision accepted for publication July 21, 1993.) 